\title{
Adaptive Modified Function Projective Synchronization of Uncertain Complex Dynamical Networks with Multiple Time-Delay Couplings and Disturbances
}

\author{
Jie Fang $(\mathbb{D}$, Na Liu, and Junwei Sun $(\mathbb{D}$ \\ College of Electric and Information Engineering, Zhengzhou University of Light Industry, Zhengzhou 450002, China \\ Correspondence should be addressed to Junwei Sun; junweisun@yeah.net
}

Received 29 September 2017; Revised 6 November 2017; Accepted 25 December 2017; Published 30 January 2018

Academic Editor: Yan-Wu Wang

Copyright (C) 2018 Jie Fang et al. This is an open access article distributed under the Creative Commons Attribution License, which permits unrestricted use, distribution, and reproduction in any medium, provided the original work is properly cited.

\begin{abstract}
This paper studies the modified function projective synchronization of uncertain complex dynamic network model with multiple time-delay couplings and external disturbances. Based on Lyapunov stability theory, the positive definite function is designed and the sufficient conditions of synchronization are given. Both the uncertain parameters and the unknown bounded disturbances are estimated in accordance with the adaptive laws. With the adaptive feedback controller, the complex dynamic network can synchronize with reference node by a scaling function matrix. The reference node can be periodic orbit, equilibrium point, or a chaotic attractor. Finally, two numerical simulations are offered to illustrate the effectiveness of the proposed method.
\end{abstract}

\section{Introduction}

Complex networks widely exist in various fields of science and engineering, ranging from biology, physics, and chemistry to social networks and technological applications. In recent years, complex dynamics networks have been an active research topic and have been developed and systematically studied [1-4] to extend nonlinear system [5]. Synchronization is a fundamental phenomenon in nature which enables the networks to achieve coherent behavior due to interaction. The research of complex network synchronization is one of the most important research directions on complex networks [68]. Up to now, many types of synchronization phenomena on complex networks have been reported, such as complete synchronization $[9,10]$, projective synchronization [11, 12], lag synchronization [13], lag projective synchronization [14], bounded synchronization [3], projective cluster synchronization [15], and global synchronization [16].

Modified function projective synchronization (MFPS) is a more general definition of synchronization method which indicates that the drive and response system could be synchronized up to a scaling function matrix. The definition of MFPS contains complete synchronization, projective synchronization, and function projective synchronization. The unpredictability of the scaling function factors in MFPS can additionally enhance the security of communication. MFPS has drawn considerable attention with a lot of research results. Reference [17] studied the MFPS of uncertain chaotic (hyperchaotic) systems based on a novel observer-based finite-time control method. Reference [18] investigated the MFPS of Liu chaotic system and its application to secure communication. References [19-21] extended the MFPS to complex domain and studied the MFPS of complex chaotic system with parameter perturbations and external perturbations.

However, the existing researches related to MFPS are mainly concerned with two chaotic systems and there are few theoretical achievements related to MFPS in a general complex network. Recently, some research results on function projective synchronization of complex dynamics networks have been achieved. Based on the adaptive open-plus-closedloop method, [22] investigated function projective synchronization of complex dynamical networks with or without external disturbances using error feedback control scheme. Based on Barbalat's lemma, [23] designed some sufficient synchronization criteria by applying the nonlinear feedback control to realize the generalized function projective synchronization between two different complex networks. Although $[22,23]$ studied the function projective synchronization of 
two complex networks, the network models are conservative for they did not take the influence of time-delay coupling into consideration. It is well known that, due to the finite speed of information transmission and processing speed among the units, the connection delays in realistic modeling of many large networks must be taken into account. What is more, there always exist some unknown factors in most real systems, covering the uncertain parameters and external disturbances which can result in network instability or poor performance. Therefore, it is significant to study the effects of time-delay coupling, uncertain parameters, and external disturbances in synchronization of complex dynamics networks. Reference [24] proposed an adaptive controller to investigate the problem of function projective synchronization in complex dynamical networks with constant time-delay coupling, uncertain parameters, and disturbance. Reference [25] investigated the modified function projective lag synchronization of dynamical complex networks with disturbance, unknown parameters, and coupling delay based on error feedback control scheme. However, the absolute constant coupling delay may be scarce in the practical networks. In [26], Du et al. achieved the function projective synchronization for general complex dynamical networks with constant or time-varying time-delay coupling by a hybrid feedback control method, but the model uncertain and external disturbances were not taken into account.

Multiple time-delay coupling complex networks indicate that there are more than one coupling delay between two nodes of complex networks. The multiple time-delay coupling complex networks widely exist in the real world such as relationship network, communication network, and transportation network. The multiple time-delay coupling complex networks can be divided into some subnetworks by different time delays [27]. The relationship network is given as an example in Figure 1. In accordance with different coupling delays, the relationship network is divided into three subnetworks. The corresponding topological structures are displayed in Figure 1. The single time-delay coupling network as a special case of multiple time-delay couplings complex networks has been studied widely $[28,29]$. However, the synchronization research of complex networks with multiple time-delay couplings is more realistic and representative, which still receives little attention.

Compared with previous work, there are three advantages which can make our research attractive and interesting. Firstly, our paper considers the multiple time-delay coupling complex networks which widely exist in the real world. The single time-delay coupling network is just a special case of multiple time-delay couplings complex networks. Secondly, MFPS is a more general synchronization method which contains complete synchronization, projective synchronization, and function projective synchronization. The unpredictability of the scaling function factors in MFPS can additionally improve the reliability of secure communication. Thirdly, our paper considers the MFPS between a complex network and a reference node. The reference node can be periodic orbit, equilibrium point, or a chaotic attractor. When the reference node is periodic orbit or equilibrium point, the idea mentioned in this paper can control complex network to a stable

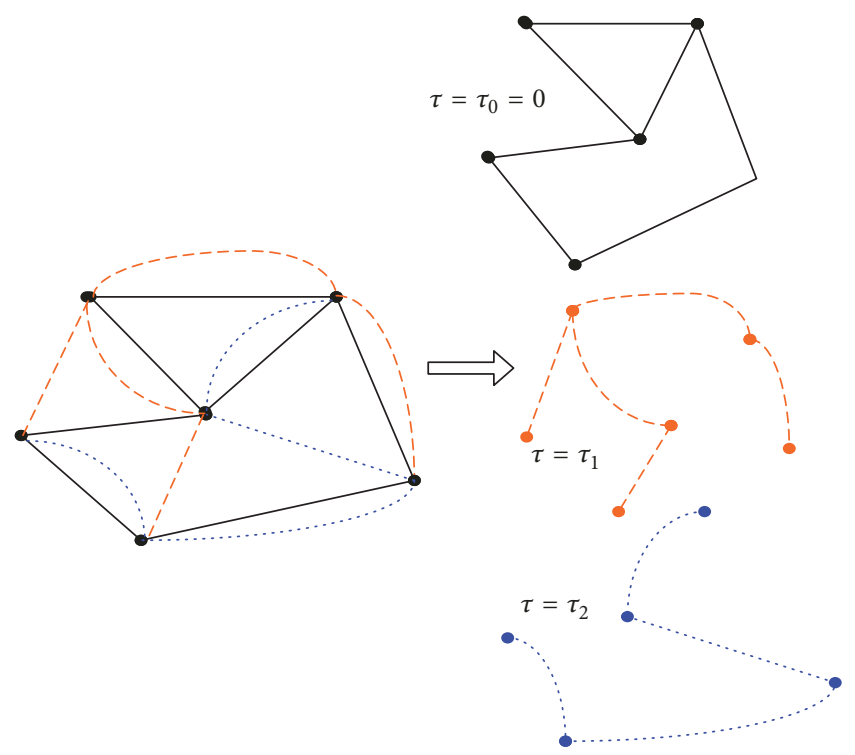

FIgURE 1: Relationship network and its division. According to different coupling delays, the relationship network is split into three subnetworks.

state. When the reference node is a chaotic attractor, the idea mentioned in this paper can synchronize complex network with a chaotic state, which can be applied in engineering fields such as secure communication and information processing.

The rest of this paper is organized as follows: the network model and some preliminaries are given in Section 2; the MFPS for uncertain complex dynamic networks with multiple time-delay couplings and disturbances is discussed in Section 3; numerical simulations are offered in Section 4; finally, the conclusive remarks are given in Section 5.

\section{Model Description and Preliminaries}

Consider a multiple time-delay coupling complex dynamical network model with unknown parameters and external disturbances as follows:

$$
\begin{aligned}
\dot{x}_{i}(t)= & f_{i}\left(x_{i}(t)\right)+F_{i}\left(x_{i}(t)\right) \theta_{i} \\
& +c \sum_{l=0}^{m-1} \sum_{j=1}^{N} a_{i j}^{l} \Gamma_{l} x_{j}\left(t-\tau_{l}(t)\right)+\Delta_{i}(t)+u_{i}(t) \\
= & f\left(x_{i}(t)\right)+F\left(x_{i}(t)\right) \theta_{i} \\
& +c \sum_{j=1}^{N} a^{0}{ }_{i j} \Gamma_{0} x_{j}\left(t-\tau_{0}(t)\right) \\
& +c \sum_{j=1}^{N} a^{1}{ }_{i j} \Gamma_{1} x_{j}\left(t-\tau_{1}(t)\right)+\cdots \\
& +c \sum_{j=1}^{N} a^{m-1}{ }_{i j} \Gamma_{m-1} x_{j}\left(t-\tau_{m-1}(t)\right)+\Delta_{i}(t) \\
& +u_{i}(t), \quad(i=1,2, \ldots, N),
\end{aligned}
$$


where $x_{i}(t)=\left(x_{i 1}(t), x_{i 2}(t), \ldots, x_{i n}(t)\right)^{T} \in R^{n}$ is the state vector of the $i$ th node; $f_{i}(\cdot): R^{n} \rightarrow R^{n}$ and $F_{i}(\cdot): R^{n} \rightarrow R^{n \times r_{i}}$ are the known continuous nonlinear function matrices; $\theta_{i} \in$ $R^{r_{i}}$ is a $r_{i}$ dimension unknown constant parameter vector. The complex network is divided into $m$ subnetworks by different coupling delays. $\tau_{l}(t) \geq 0,(l=0,1,2, \ldots, m-1)$ denotes different coupling delays which can be constant time delays or time-varying delays, and especially $\tau_{0}(t)=0$ means that the coupling delay is $0 . \Delta_{i} \in R^{n}$ is the disturbance; $u_{i}(t) \in R^{n}$ is the control input; $c$ is the coupling strength; $\Gamma_{l}$ is the innercoupling matrices which describes the individual couplings between nodes $i$ and $j ; A_{l}=\left(a_{i j}^{l}\right)_{N \times N}, l=0,1,2, \ldots, m-$ 1 is the weight configuration matrices, representing the topological structure of the network. If nodes $i$ and $j$ have a connection, then $a_{i j}^{l}=a_{j i}^{l} \neq 0,(i \neq j)$, otherwise, $a_{i j}^{l}=a_{j i}^{l}=0,(i \neq j)$, and the diagonal elements of matrix $A_{l}$ are defined as

$$
a_{i i}^{l}=-\sum_{j=1, j \neq i}^{N} a_{i j}^{l}, \quad i=1,2, \ldots, N
$$

Definition 1 (MFPS). For the uncertain complex dynamic network model with multiple time-delay couplings and external disturbances, it is said that model (1) and reference node $s(t)$ realize MFPS if there exists a continuously differentiable scaling function matrix $H(t)=\operatorname{diag}\left(h_{1}(t), h_{2}(t), \ldots, h_{n}(t)\right)$, such that

$$
\begin{aligned}
\lim _{t \rightarrow \infty}\left\|\mathbf{e}_{i}(t)\right\|=\lim _{t \rightarrow \infty}\left\|x_{i}(t)-H(t) s(t)\right\| & =0, \\
& i=1,2, \ldots, N,
\end{aligned}
$$

where $\|\cdot\|$ denotes the Euclidean norm of a vector. $s(t) \in R^{n}$ is the state vector of an isolated node and satisfies $\dot{s}(t)=g(s(t))$. $s(t)$ can be periodic orbit, equilibrium point, or a chaotic attractor.

Assumption 2. The external disturbance $\Delta_{i}(t)$ is bounded, and there exists a positive constant $d_{i}>0$, such that $\left\|\Delta_{i}\right\| \leq$ $d_{i}, i=1,2, \ldots, N$.

Assumption 3. $\tau_{l}(t), l=0,1, \ldots, m-1$, is a differentiable function with $0 \leq \dot{\tau}_{l}(t) \leq \varepsilon<1$. Clearly, this assumption is satisfied if $\tau_{l}(t)$ is a constant.

Lemma 4 (see [25]). For any vectors $X, Y \in R^{n}$ and a positive definite matrix $Q \in R^{n \times n}$, the following matrix inequality holds: $2 X^{T} Q Y \leq X^{T} Q Q^{T} X+Y^{T} Y$.

\section{MFPS Synchronization Scheme}

Theorem 5. For a given synchronization scaling function matrix $H(t)$ and any initial conditions $x_{i}(0)$ and $s(0)$, if Assumptions 2 and 3 are satisfied, the uncertain complex dynamic network model (1) and the isolated node $s(t)$ will realize MFPS with the following adaptive control laws:

$$
\begin{aligned}
& \dot{\hat{\theta}}_{i}=k_{1} F_{i}^{T}\left(x_{i}(t)\right) e_{i}, \\
& \dot{\hat{d}}_{i}=k_{2} e_{i}^{T} \operatorname{sign}\left(e_{i}\right), \\
& \dot{\hat{q}}_{i}=k_{3} e_{i}^{T} e_{i},
\end{aligned}
$$$$
u_{i}(t)=-f_{i}\left(x_{i}(t)\right)+\dot{H}(t) s(t)+H(t) \dot{s}(t)
$$$$
-F_{i}\left(x_{i}(t)\right) \widehat{\theta}_{i}-\widehat{d}_{i} \operatorname{sign}\left(e_{i}\right)-\widehat{q}_{i} e_{i}(t),
$$$$
(i=1,2, \ldots, N) \text {, }
$$

where $k_{i}>0, i=1,2,3$, are three positive constants; $\hat{\theta}_{i}$ is the estimated parameter for $\theta_{i} ; \hat{d}_{i}$ is the estimated parameter for $d_{i}$; $\widehat{q}_{i}$ is adaptive feedback control gains; $\operatorname{sign}(\cdot)$ is the sign function.

Proof. From Definition 1, we have the error term

$$
\mathbf{e}_{i}(t)=x_{i}(t)-H(t) s(t) \quad(i=1,2, \ldots, N) .
$$

The time derivative of $(8)$ is

$$
\begin{aligned}
\dot{e}_{i}(t)=\dot{x}_{i}(t)-H(t) \dot{s}(t)-\dot{H}(t) s(t) & \\
& (i=1,2, \ldots, N) .
\end{aligned}
$$

Substituting (1) into (9), we have

$$
\begin{aligned}
\dot{e}_{i}(t)= & f_{i}\left(x_{i}(t)\right)+F_{i}\left(x_{i}(t)\right) \theta_{i} \\
& +c \sum_{l=0}^{m-1} \sum_{j=1}^{N} a_{i j}^{l} \Gamma_{l} x_{j}\left(t-\tau_{l}(t)\right)+\Delta_{i}(t)+u_{i}(t) \\
& -H(t) \dot{s}(t)-\dot{H}(t) s(t) \\
= & f_{i}\left(x_{i}(t)\right)+F_{i}\left(x_{i}(t)\right) \theta_{i}+c \sum_{j=1}^{N} a^{0}{ }_{i j} \Gamma_{0} x_{j}(t) \\
& +c \sum_{l=1}^{m-1} \sum_{j=1}^{N} a^{l}{ }_{i j} \Gamma_{l} x_{j}\left(t-\tau_{l}(t)\right)+\Delta_{i}(t)+u_{i}(t) \\
& -H(t) \dot{s}(t)-\dot{H}(t) s(t) .
\end{aligned}
$$

Substituting (4) into (10), we have

$$
\begin{aligned}
\dot{e}_{i}(t)= & F_{i}\left(x_{i}(t)\right)\left(\theta_{i}-\widehat{\theta}_{i}\right)+c \sum_{j=1}^{N} a^{0}{ }_{i j} \Gamma_{0} x_{j}(t) \\
& +c \sum_{l=1}^{m-1} \sum_{j=1}^{N} a_{i j}^{l} \Gamma_{l} x_{j}\left(t-\tau_{l}(t)\right)+\Delta_{i}(t) \\
& -\widehat{d}_{i} \operatorname{sign}\left(e_{i}\right)-\widehat{q}_{i} e_{i}(t) .
\end{aligned}
$$


Choose the following Lyapunov function:

$$
\begin{aligned}
V(t)= & \frac{1}{2} \sum_{i=1}^{N} e_{i}(t)^{T} e_{i}(t) \\
& +\frac{1}{2(1-\varepsilon)} \int_{t-\tau_{l}(t)}^{t} \sum_{l=1}^{m-1} \sum_{i=1}^{N} e_{i}^{T}(\delta) e_{i}(\delta) d \delta \\
& +\frac{1}{2 k_{1}} \sum_{i=1}^{N} \widetilde{\theta}_{i}^{T} \widetilde{\theta}_{i}+\frac{1}{2 k_{2}} \sum_{i=1}^{N} \widetilde{d}_{i}^{2} \\
& +\frac{1}{2 k_{3}} \sum_{i=1}^{N}\left(\widehat{q}_{i}-q^{*}\right)^{2},
\end{aligned}
$$

where $\widetilde{\theta}_{i}=\widehat{\theta}_{i}-\theta_{i}, \widetilde{d}_{i}=\widehat{d}_{i}-d_{i}, 0<\varepsilon<1$, and $q^{*}$ is the positive constants to be designed later.

The time derivative of $V(t)$ is

$$
\begin{aligned}
\dot{V}(t)= & \sum_{i=1}^{N} e_{i}^{T}(t) \dot{e}_{i}(t)+\frac{1}{2(1-\varepsilon)} \sum_{l=1}^{m-1} \sum_{i=1}^{N} e_{i}^{T}(t) e_{i}(t) \\
& -\frac{1-\dot{\tau}_{l}(t)}{2(1-\varepsilon)} \sum_{l=1}^{m-1} \sum_{i=1}^{N} e_{i}^{T}\left(t-\tau_{l}(t)\right) e_{i}\left(t-\tau_{l}(t)\right) \\
& +\frac{1}{k_{1}} \sum_{i=1}^{N} \dot{\hat{\theta}}_{i}^{T} \tilde{\theta}_{i}+\frac{1}{k_{2}} \sum_{i=1}^{N}\left(\widehat{d}_{i}-d_{i}\right) \dot{\hat{d}}_{i} \\
& +\frac{1}{k_{3}} \sum_{i=1}^{N}\left(\widehat{q}_{i}-q^{*}\right) \dot{\hat{q}}_{i} .
\end{aligned}
$$

Substituting (11) into (13), we have

$$
\begin{aligned}
& \dot{V}(t)=\sum_{i=1}^{N} e_{i}^{T}(t)\left[-F_{i}\left(x_{i}(t)\right) \tilde{\theta}_{i}+c \sum_{j=1}^{N} a^{0}{ }_{i j} \Gamma_{0} e_{j}(t)\right. \\
& +c \sum_{l=1}^{m-1} \sum_{j=1}^{N} a^{l}{ }_{i j} \Gamma_{l} e_{j}\left(t-\tau_{l}(t)\right)+\Delta_{i}(t)-\widehat{d}_{i} \operatorname{sign}\left(e_{i}\right) \\
& \left.-\widehat{q}_{i} e_{i}(t)\right]+\frac{1}{2(1-\varepsilon)} \sum_{l=1}^{m-1} \sum_{i=1}^{N} e_{i}^{T}(t) e_{i}(t) \\
& -\frac{1-\dot{\tau}_{l}(t)}{2(1-\varepsilon)} \sum_{l=1}^{m-1} \sum_{i=1}^{N} e_{i}^{T}\left(t-\tau_{l}(t)\right) e_{i}\left(t-\tau_{l}(t)\right)+\frac{1}{k_{1}} \\
& \quad \sum_{i=1}^{N} \dot{\hat{\theta}}_{i}^{T} \tilde{\theta}_{i}+\frac{1}{k_{2}} \sum_{i=1}^{N}\left(\hat{d}_{i}-d_{i}\right) \dot{\hat{d}}_{i}+\frac{1}{k_{3}} \sum_{i=1}^{N}\left(\widehat{q}_{i}-q^{*}\right) \dot{\hat{q}}_{i} .
\end{aligned}
$$

Substituting (5), (6), and (7) into (14), we have

$$
\begin{aligned}
& \dot{V}(t)=\sum_{i=1}^{N} e_{i}^{T}(t)\left[c \sum_{j=1}^{N} a^{0}{ }_{i j} \Gamma_{0} e_{j}(t)\right. \\
& \left.+c \sum_{l=1}^{m-1} \sum_{j=1}^{N} a^{l}{ }_{i j} \Gamma_{l} e_{j}\left(t-\tau_{l}(t)\right)+\Delta_{i}(t)\right]+\frac{1}{2(1-\varepsilon)}
\end{aligned}
$$

$$
\begin{aligned}
& \cdot \sum_{l=1}^{m-1} \sum_{i=1}^{N} e_{i}^{T}(t) e_{i}(t)-\frac{1-\dot{\tau}_{l}(t)}{2(1-\varepsilon)} \\
& \cdot \sum_{l=1}^{m-1} \sum_{i=1}^{N} e_{i}^{T}\left(t-\tau_{l}(t)\right) e_{i}\left(t-\tau_{l}(t)\right) \\
& -\sum_{i=1}^{N} d_{i} e_{i}^{T} \operatorname{sign}\left(e_{i}\right)-\sum_{i=1}^{N} q^{*} e_{i}^{T} e_{i}
\end{aligned}
$$

Let $e(t)=\left(e_{1}^{T}(t), e_{2}^{T}(t), \ldots, e_{N}^{T}(t)\right)^{T} \in R^{n N}$ and $P_{0}=\left(A_{0} \otimes\right.$ $\left.\Gamma_{0}\right), P_{1}=\left(A_{1} \otimes \Gamma_{1}\right), \ldots, P_{l}=\left(A_{l} \otimes \Gamma_{l}\right)$, where $\otimes$ represents the Kronecker product.

$$
\begin{aligned}
\dot{V}(t)= & c e^{T}(t) P_{0} e(t)+c \sum_{l=1}^{m-1} e^{T}(t) P_{l} e\left(t-\tau_{l}(t)\right) \\
& +\frac{1}{2(1-\varepsilon)} \sum_{l=1}^{m-1} e^{T}(t) e(t) \\
& -\frac{1-\dot{\tau}_{l}(t)}{2(1-\varepsilon)} \sum_{l=1}^{m-1} e^{T}(t-\tau(t)) e(t-\tau(t)) \\
& +\sum_{i=1}^{N} e_{i}^{T}(t) \Delta_{i}(t)-\sum_{i=1}^{N} d_{i} e_{i}^{T} \operatorname{sign}\left(e_{i}\right) \\
& -q^{*} e^{T}(t) e(t) .
\end{aligned}
$$

By Assumptions 2 and 3, we have $1 / 2 \leq(1-\dot{\tau}(t)) / 2(1-\varepsilon)$, $e_{i}^{T} \Delta_{i}(t) \leq d_{i} e_{i}^{T} \operatorname{sign}\left(e_{i}\right)$, so

$$
\begin{aligned}
\dot{V}(t) \leq & c e^{T}(t) P_{0} e(t)+c \sum_{l=1}^{m-1} e^{T}(t) P_{l} e\left(t-\tau_{l}(t)\right) \\
& +\frac{1}{2(1-\varepsilon)} \sum_{l=1}^{m-1} e^{T}(t) e(t) \\
& -\frac{1}{2} \sum_{l=1}^{m-1} e^{T}(t-\tau(t)) e(t-\tau(t)) \\
& -q^{*} e^{T}(t) e(t) .
\end{aligned}
$$

By Lemma 4, we have $c e^{T}(t) P_{l} e\left(t-\tau_{l}(t)\right) \leq$ 2) $c^{2} e^{T}(t) P_{l} P_{l}^{T} e(t)+(1 / 2) e^{T}(t-\tau(t)) e(t-\tau(t))$, so

$$
\begin{aligned}
\dot{V}(t) & \leq e^{T}(t)\left[c P_{0}+\frac{1}{2} c^{2} \sum_{l=1}^{m-1} P_{l} P_{l}^{T}\right] e(t) \\
+ & \sum_{l=1}^{m-1} \frac{1}{2(1-\varepsilon)} e^{T}(t) e(t)-q^{*} e^{T}(t) e(t) \\
& \leq\left[\lambda_{\max }\left(c P_{0}+\frac{1}{2} \sum_{l=1}^{m-1} c^{2} P_{l} P_{l}^{T}\right)+\frac{m-1}{2(1-\varepsilon)}-q^{*}\right] \\
\cdot & e^{T}(t) e(t),
\end{aligned}
$$


where $\lambda_{\max }(Q)$ is the largest eigenvalue of Matrix $Q$. Therefore, by taking appropriate $q^{*}$ such that

$$
q^{*} \geq \lambda_{\max }\left(c P_{0}+\frac{1}{2} \sum_{l=1}^{m-1} c^{2} P_{l} P_{l}^{T}\right)+\frac{m-1}{2(1-\varepsilon)},
$$

based on the LaSalle invariance principle, we can obtain $\dot{V}(t) \leq 0$. According to Lyapunov-Krasovskii stable theorem, we can obtain $e_{i}(t) \rightarrow 0$ as $t \rightarrow \infty$, which means that the MFPS between network (1) and reference node $s(t)$ is achieved with control input (4) and updated laws (5)-(7). This completes the proof.

Remark 6. In this paper, the multiple time-delay couplings $\tau_{l}(t)>0,(l=0,1,2, \ldots, m-1)$ can be constant time delay or time-varying time delay. When the time delay $\tau_{l}(t)$ is constant, that is, $\dot{\tau}_{l}(t)=0$, it also satisfies Assumption 3, so controller (4) and adaptive laws (5)-(7) are also practical.

Remark 7. If the parameter $\theta_{i}, i=1,2, \ldots, n$, is known, for given scaling function matrix $H(t)$, the complex dynamics networks (1) and reference node $s(t)$ can realize MFPS by the following controller and adaptive laws:

$$
\begin{aligned}
u_{i}(t)= & -f_{i}\left(x_{i}(t)\right)+\dot{H}(t) s(t)+H(t) \dot{s}(t) \\
& -F_{i}\left(x_{i}(t)\right) \theta_{i}-\widehat{d}_{i} \operatorname{sign}\left(e_{i}\right)-\widehat{q}_{i} e_{i}(t), \\
\dot{\vec{d}}_{i}= & k_{2} e_{i}^{T} \operatorname{sign}\left(e_{i}\right), \\
\dot{\hat{q}}_{i}= & k_{3} e_{i}^{T} e_{i} \\
& (i=1,2, \ldots, N) .
\end{aligned}
$$

Remark 8. If $m_{1}(t)=m_{2}(t)=\cdots=m_{n}(t)=m(t)$ or $m_{1}(t)=$ $m_{2}(t)=\cdots=m_{n}(t)=m$, the MFPS scheme in this paper can also extend to solve function projective synchronization or projective synchronization problems of complex networks with multiple time-delay couplings.

Remark 9. In a lot of literature about complex network synchronization, the controller always includes the time delay $\tau(t)$. However, it is difficult to measure the delay and implement the delay term, especially the time-varying delay term in the real control system. In our work, the controller does not include $\tau(t)$, so the proposed method is more general and realistic.

\section{Illustrative Examples}

In this section, we will study the MFPS of the uncertain complex dynamical networks with multiple time-delay couplings and disturbances. We take the Lü chaotic system as reference node to verify the effectiveness of the proposed method. The Lü system is described as follows:

$$
\begin{aligned}
& \dot{s}_{1}=a\left(s_{2}-s_{1}\right), \\
& \dot{s}_{2}=b s_{2}-s_{1} s_{3}, \\
& \dot{s}_{3}=s_{1} s_{2}-c s_{3},
\end{aligned}
$$

where $s_{1}, s_{2}, s_{3}$ are the state variables and $a, b, c$ are real constants. When $\alpha=36, b=20, c=3$, the system is chaotic attractor, which is shown in Figure 2.

Example 1. Consider a coupled complex dynamical network consisting of six Lorenz chaotic systems and two different constant time-delay couplings, that is, $N=6, m=3$. The topological structure matrices $A_{0}, A_{1}, A_{2}$ are as follows:

$$
\begin{aligned}
A_{0}= & \left(\begin{array}{cccccc}
-3 & 1 & 0 & 1 & 1 & 0 \\
1 & -2 & 0 & 0 & 1 & 1 \\
0 & 0 & -3 & 0 & 0 & 1 \\
1 & 0 & 0 & -2 & 0 & 0 \\
1 & 1 & 0 & 0 & -4 & 1 \\
0 & 1 & 1 & 0 & 1 & -1
\end{array}\right), \\
A_{1}= & \left(\begin{array}{cccccc}
-1 & 1 & 0 & 0 & 0 & 1 \\
1 & -2 & 1 & 0 & 0 & 0 \\
0 & 1 & -1 & 0 & 1 & 0 \\
0 & 0 & 0 & -3 & 1 & 1 \\
0 & 0 & 1 & 1 & -2 & 1 \\
1 & 0 & 0 & 1 & 1 & -2
\end{array}\right), \\
A_{2}= & \left(\begin{array}{cccccc}
-3 & 0 & 0 & 1 & 1 & 0 \\
0 & -1 & 1 & 0 & 1 & 1 \\
0 & 1 & -2 & 0 & 0 & 0 \\
1 & 0 & 0 & -3 & 0 & 0 \\
1 & 1 & 0 & 0 & -1 & 1 \\
0 & 1 & 0 & 0 & 1 & -2
\end{array}\right),
\end{aligned}
$$

where matrix $A_{0}$ has no time delay and matrices $A_{1}$ and $A_{2}$ have different kinds of constant time delays.

Through the above analysis, the network model can be written as

$$
\begin{aligned}
{\left[\begin{array}{l}
x_{i 1}(t) \\
x_{i 2}(t) \\
x_{i 3}(t)
\end{array}\right]=} & {\left[\begin{array}{c}
0 \\
-x_{i 1}(t) x_{i 3}(t)-x_{i 2}(t) \\
x_{i 1}(t) x_{i 2}(t)
\end{array}\right] } \\
& +\left[\begin{array}{ccc}
x_{i 2}(t)-x_{i 1}(t) & 0 & 0 \\
0 & x_{i 1}(t) & 0 \\
0 & 0 & -x_{i 3}
\end{array}\right]\left[\begin{array}{c}
\theta_{i 1} \\
\theta_{i 2} \\
\theta_{i 3}
\end{array}\right] \\
& +c \sum_{j=1}^{6} a^{0}{ }_{i j} \Gamma_{0} x_{j}(t)+c \sum_{j=1}^{6} a^{1}{ }_{i j} \Gamma_{1} x_{j}\left(t-\tau_{1}\right) \\
& +c \sum_{j=1}^{6} a^{2}{ }_{i j} \Gamma_{2} x_{j}\left(t-\tau_{2}\right)+\Delta_{i}(t)+u_{i}(t),
\end{aligned}
$$

where $i=1,2, \ldots, 5,6$, and the true value for each parameter is $\theta_{i}=\left(\theta_{i 1}, \theta_{i 2}, \theta_{i 2}\right)^{T}=(10,28,8 / 3)^{T}$. 

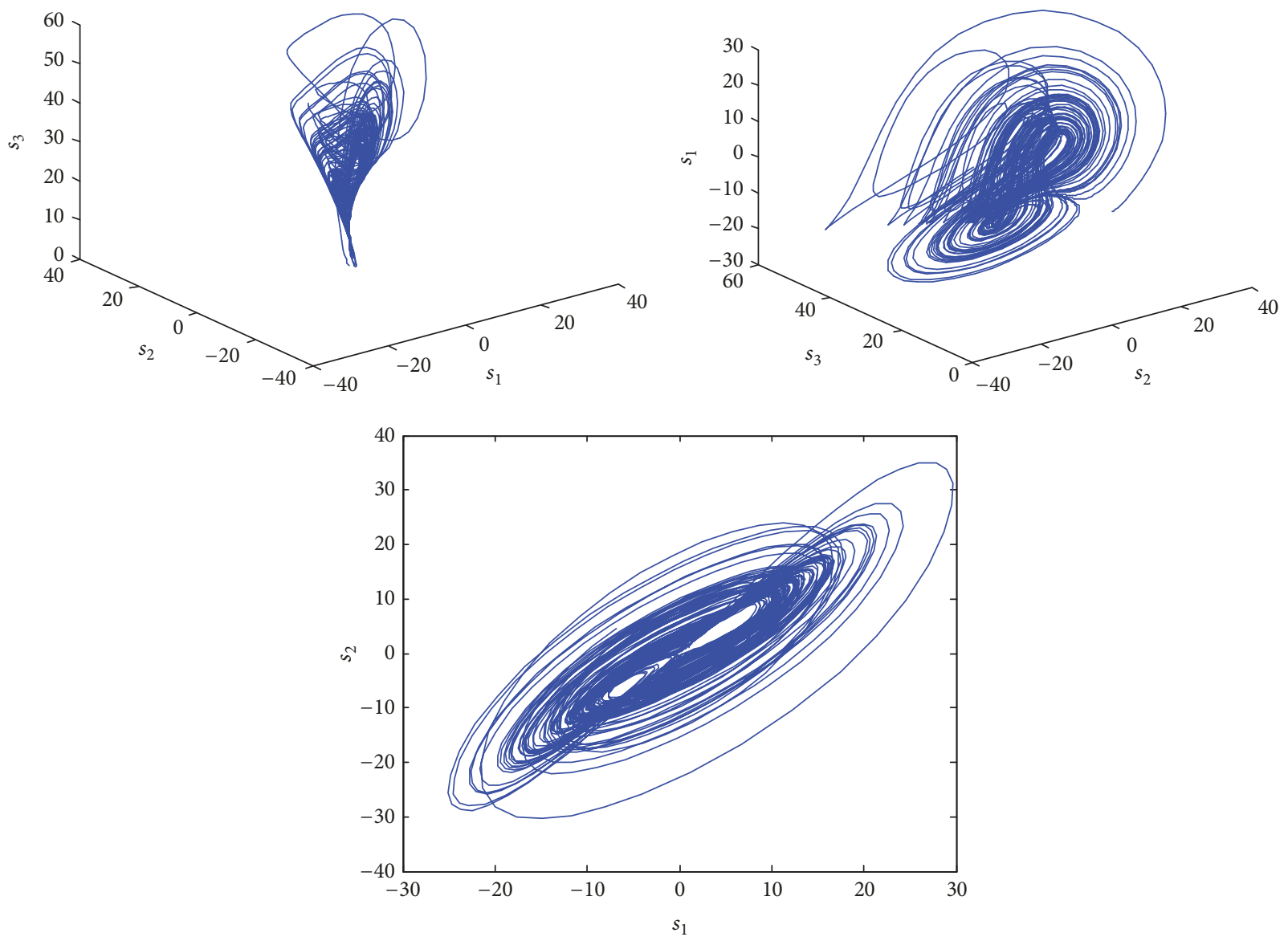

FIGURE 2: 2D and 3D projections of chaotic attractor of the Lü system.

In the numerical simulations, we set $c=3, \tau_{1}=$ $0.05 \mathrm{~s}, \tau_{2}=0.1 \mathrm{~s}$ and network inner-coupling matrix $\Gamma_{0}=$ $\Gamma_{1}=\Gamma_{2}=I_{3 \times 3}$. Choose the disturbance $\Delta_{d}=[0.3 \cos (t)$, $0.2 \sin (t), 0.5 \sin (t)]$, the scaling function matrix $H(t)=$ $\operatorname{diag}(2+\sin (\pi t / 5), 3-\cos (\pi t), 3+\sin (2 \pi t / 10)), k_{1}=4, k_{2}=8$, $k_{3}=5$. The simulation results are showed in Figures 3-6. Figure 3 displays the time evolution of the synchronization errors. Figure 4 displays the estimated parameters $\theta_{i 1}, \theta_{i 2}$, $\theta_{i 3}$. The estimated parameter for external disturbances $d_{i}$ is depicted in Figure 5. The adaptive feedback gain $q_{i}$ is depicted in Figure 6. These results show that the MFPS takes place between the complex dynamical networks and the reference note $s(t)$. What is more, all the uncertain parameters are identified successfully by the parameter adaptive laws.

Example 2. Consider a coupled complex dynamical network consisting of four nodes with two time-delay couplings, that is, $N=4, m=3$. The network model can be written as

$$
\begin{aligned}
{\left[\begin{array}{c}
x_{i 1}(t) \\
x_{i 2}(t) \\
x_{i 3}(t)
\end{array}\right]=} & {\left[\begin{array}{c}
0 \\
-x_{i 1}(t) x_{i 3}(t)-x_{i 2}(t) \\
x_{i 1}(t) x_{i 2}(t)
\end{array}\right] } \\
& +\left[\begin{array}{ccc}
x_{i 2}(t)-x_{i 1}(t) & 0 & 0 \\
0 & x_{i 1}(t) & 0 \\
0 & 0 & -x_{i 3}
\end{array}\right]\left[\begin{array}{c}
\theta_{i 1} \\
\theta_{i 2} \\
\theta_{i 3}
\end{array}\right]
\end{aligned}
$$

$$
\begin{aligned}
& +c \sum_{j=1}^{6} a^{0}{ }_{i j} \Gamma_{0} x_{j}(t)+c \sum_{j=1}^{6} a^{1}{ }_{i j} \Gamma_{1} x_{j}\left(t-\tau_{1}\right) \\
& +c \sum_{j=1}^{6} a^{2}{ }_{i j} \Gamma_{2} x_{j}\left(t-\tau_{2}\right)+\Delta_{i}(t)+u_{i}(t),
\end{aligned}
$$

where $i=1,2,3,4$ and $\tau_{1}(t)=0.6 \mathrm{~s}$ is a constant time delay. $\tau_{2}(t)=e^{t} /\left(2+e^{t}\right)$ is a time-varying delay and then $\dot{\tau}_{2}(t)=$ $2 e^{t} /\left(2+e^{t}\right)^{2} \in(0,1 / 2]$. The topological structure matrices $A_{0}, A_{1}, A_{2}$ are as follows:

$$
\begin{aligned}
A_{0} & =\left(\begin{array}{cccc}
-1 & 1 & 0 & 0 \\
1 & 0 & 1 & 0 \\
0 & 1 & -1 & 0 \\
0 & 0 & 0 & -2
\end{array}\right), \\
A_{1} & =\left(\begin{array}{cccc}
-2 & 1 & 1 & 0 \\
1 & -3 & 1 & 0 \\
1 & 1 & -4 & 1 \\
0 & 0 & 1 & -1
\end{array}\right),
\end{aligned}
$$



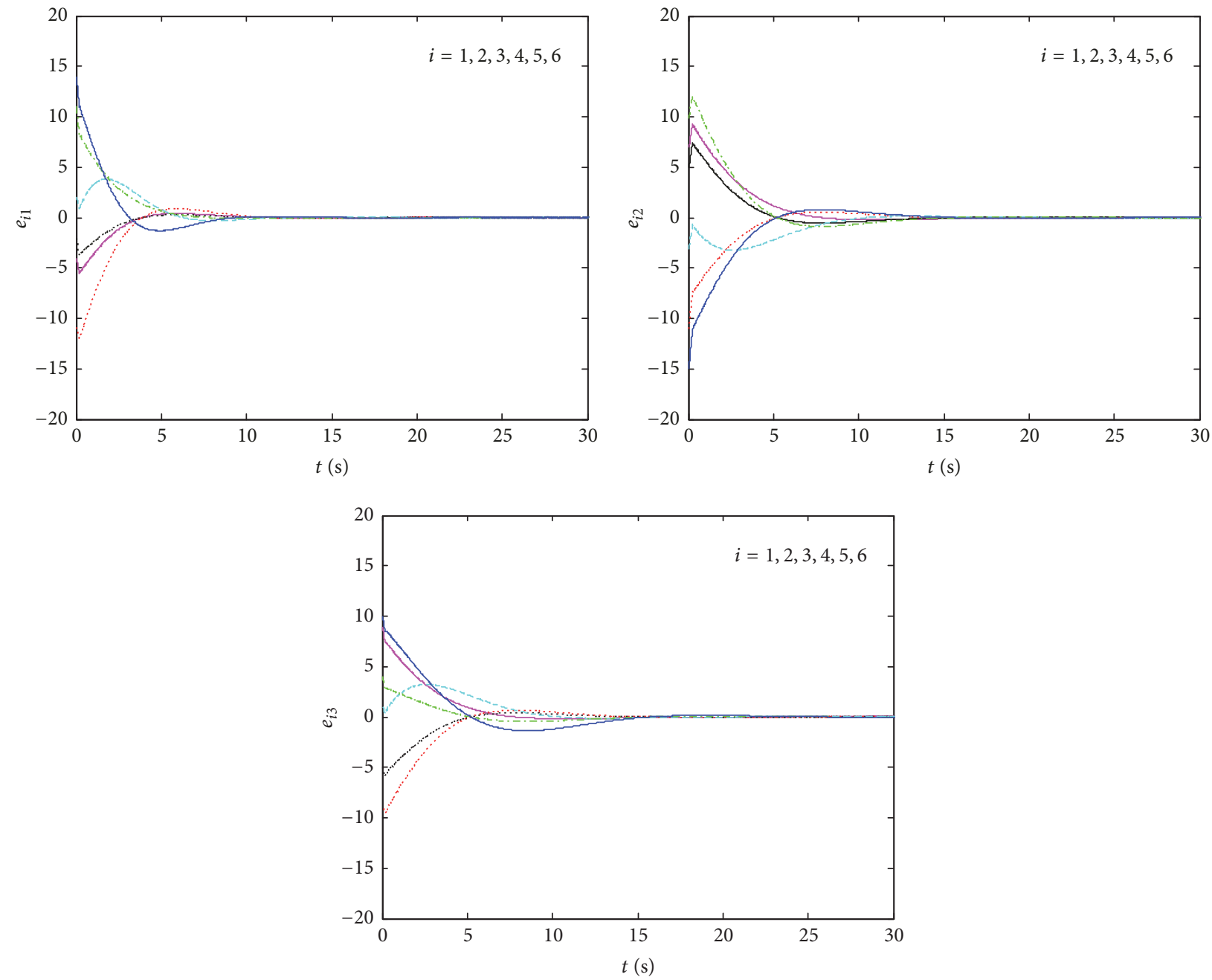

FIgURE 3: The time evolution of synchronization errors $e_{i 1}, e_{i 2}, e_{i 3}$ with constant time delay $(N=6, m=3)$.

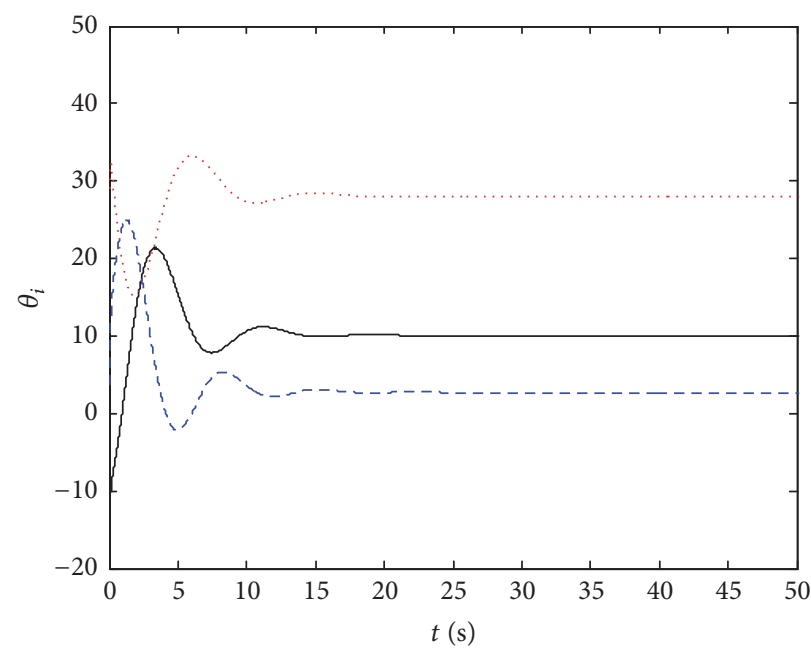

$$
\begin{array}{ll} 
& \theta_{i 1} \\
\cdots \cdots & \theta_{i 2} \\
--- & \theta_{i 3}
\end{array}
$$

FIgURE 4: The estimation of the unknown parameters $\theta_{i 1}, \theta_{i 2}, \theta_{i 3}$ with constant time delay $(N=6, m=3)$. 


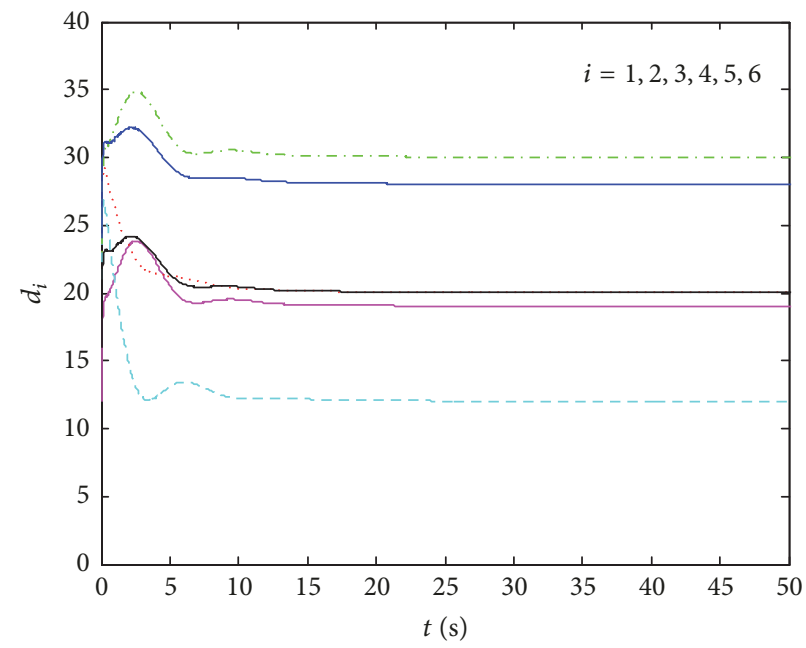

Figure 5: The time evolution of the disturbance parameters $d_{i}$ with constant time delay $(N=6, m=3)$.

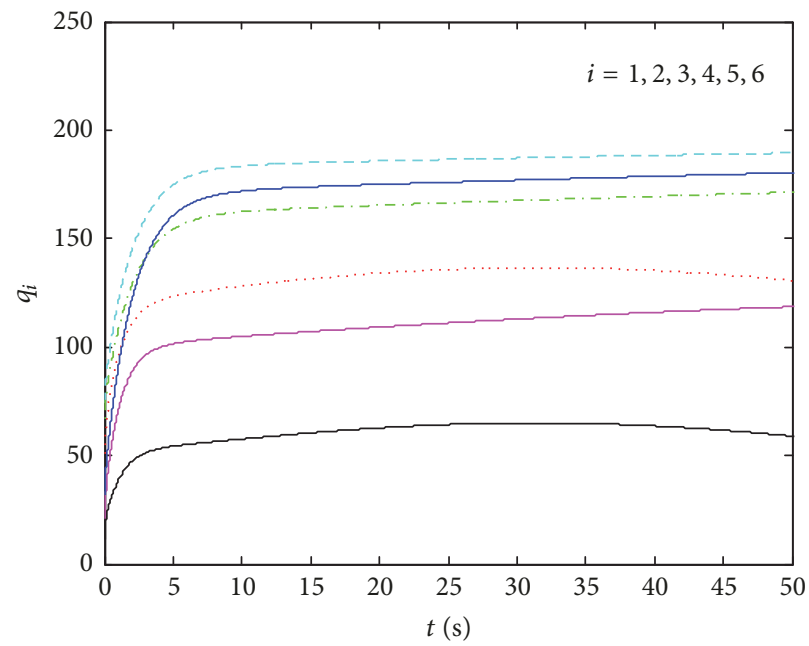

FIGURE 6: The time evolution of feedback gains $q_{i}$ with constant time delay $(N=6, m=3)$.

$$
A_{2}=\left(\begin{array}{cccc}
-2 & 0 & 0 & 0 \\
0 & -3 & 1 & 1 \\
0 & 1 & 0 & 0 \\
0 & 1 & 0 & -2
\end{array}\right)
$$

Similar to Example 1, the simulation results are given in Figures 7-10, and the numerical results are given to show that the theoretical results are also effective to the complex dynamical networks with multiple time-varying delay couplings.

\section{Conclusion}

This paper studied the MFPS of uncertain complex dynamic network with multiple time-delay couplings and external disturbances. The complex networks can be divided into some subnetworks by different coupling delays. Through the adaptive feedback controller, the complex network can synchronize with reference node according to the expected scaling function matrix. The uncertain parameters, feedback gains, and bounds of the external disturbances are all estimated by the adaptive laws. The controller does not include the delay term, so the proposed method is more general and realistic. The corresponding theoretical proofs and numerical simulations are given to demonstrate the validity and feasibility of the proposed control technique. The idea may be applied in engineering fields such as secure communication and information processing. How to realize MFPS of uncertain complex dynamical networks with multiple time-delay couplings in actual practice is our next research topic.

\section{Conflicts of Interest}

The authors declare that they have no conflicts of interest. 

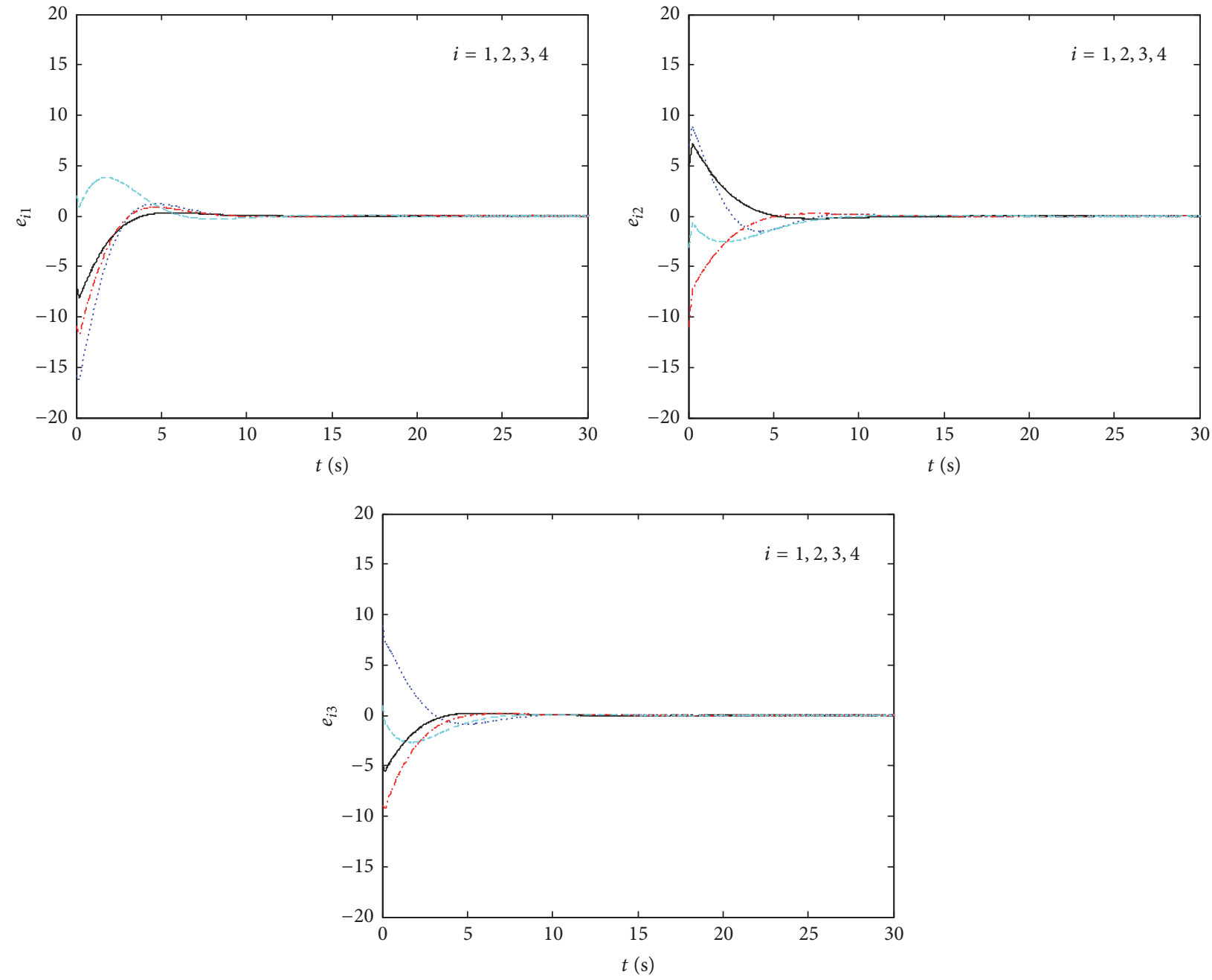

FIGURE 7: The time evolution of synchronization errors $e_{i 1}, e_{i 2}, e_{i 3}$ with time-varying delay $(N=4, m=3)$.

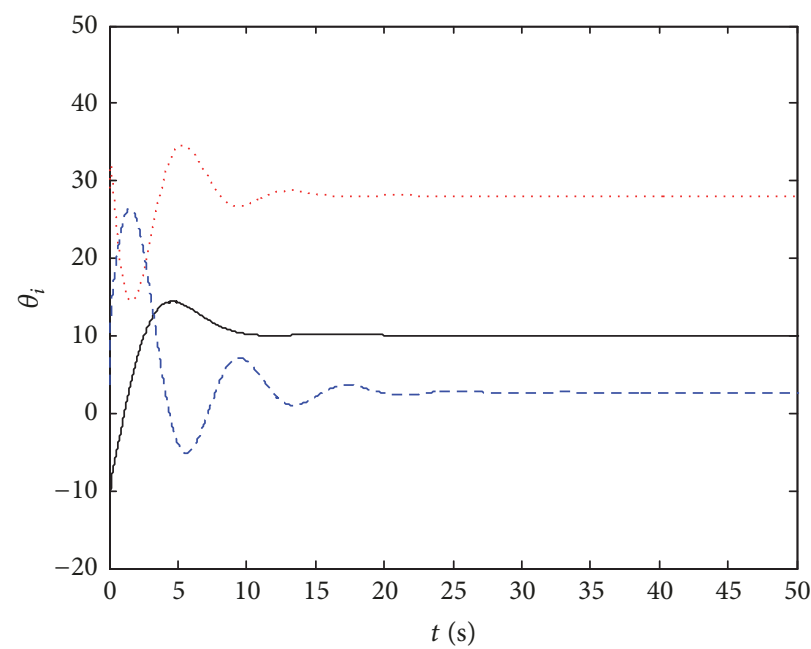

$$
\begin{aligned}
& -\theta_{i 1} \\
& \cdots \cdots \theta_{i 2} \\
& ---\theta_{i 3}
\end{aligned}
$$

FIGURE 8: The estimation of the unknown parameters $\theta_{i 1}, \theta_{i 2}, \theta_{i 3}$ with time-varying delay $(N=4, m=3)$. 


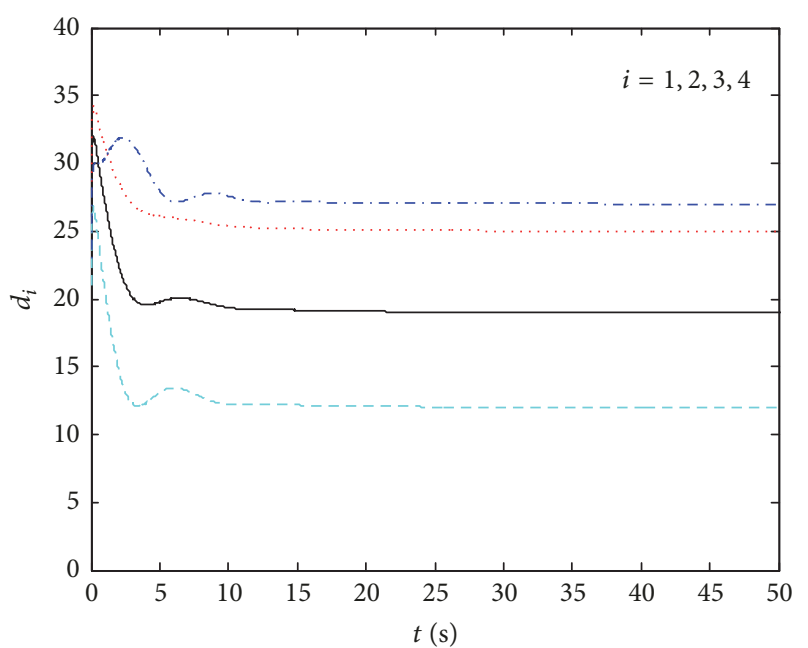

Figure 9: The time evolution of the disturbance parameters $d_{i}$ with time-varying delay $(N=4, m=3)$.

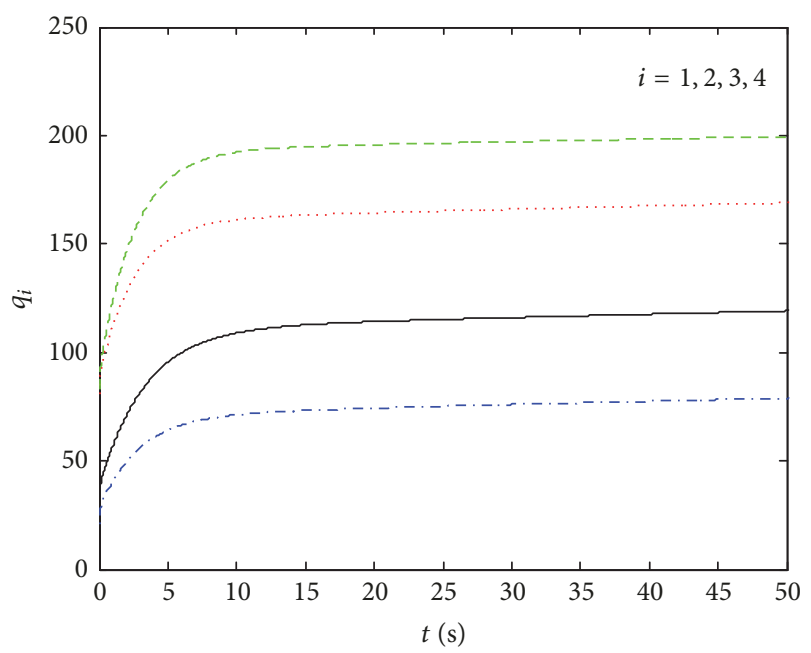

FIGURE 10: The time evolution of feedback gains $q_{i}$ with time-varying delay $(N=4, m=3)$.

\section{Acknowledgments}

The work is supported by the National Natural Science Foundation of China (Grant nos. 61775198 and 61603348), the Henan Province Natural Science Foundation (Grant no. 162300410323), the Research Program of Henan Province (Grant nos. 15IRTSTHN012, 162300410220, and 17A120005), and the Henan Province Young-Backbone Teacher Foundation (Grant no. 2016GGJS090).

\section{References}

[1] S. H. Strogatz, "Exploring complex networks," Nature, vol. 410, no. 6825 , pp. 268-276, 2001.

[2] J. H. Lü, "Mathematical models and synchronization criterions of complex dynamical networks," Systems Engineering-Theory and Practice, vol. 24, no. 4, pp. 17-22, 2004.

[3] Y. Xu, Y. Lu, W. Yan, W. Zhou, and J. Fang, "Bounded synchronization of the general complex dynamical network with delay feedback controller," Nonlinear Dynamics, vol. 84, no. 2, pp. 661667, 2016.

[4] J. Sun, Y. Wu, G. Cui, and Y. Wang, "Finite-time real combination synchronization of three complex-variable chaotic systems with unknown parameters via sliding mode control," Nonlinear Dynamics, vol. 88, no. 3, pp. 1677-1690, 2017.

[5] J. W. Sun and Y. Shen, "Quasi-ideal memory system," IEEE Transactions on Cybernetics, vol. 45, no. 7, pp. 1353-1362, 2015.

[6] Y. Xu, W. Zhou, J. Fang, C. Xie, and D. Tong, "Finite-time synchronization of the complex dynamical network with nonderivative and derivative coupling," Neurocomputing, vol. 173, pp. 1356-1361, 2016.

[7] F. Dörfler and F. Bullo, "Synchronization in complex networks of phase oscillators: A survey," Automatica, vol. 50, no. 6, pp. 1539-1564, 2014.

[8] J. Sun, G. Cui, Y. Wang, and Y. Shen, "Combination complex synchronization of three chaotic complex systems," Nonlinear Dynamics, vol. 79, no. 2, pp. 953-965, 2014.

[9] H. Zhao, L. Li, H. Peng, J. Xiao, Y. Yang, and M. Zheng, "Impulsive control for synchronization and parameters identification of uncertain multi-links complex network," Nonlinear Dynamics, vol. 83, no. 3, pp. 1437-1451, 2016.

[10] J. Sun, Y. Wang, Y. Wang, and Y. Shen, "Finite-time synchronization between two complex-variable chaotic systems with unknown parameters via nonsingular terminal sliding mode control," Nonlinear Dynamics, vol. 85, no. 2, pp. 1105-1117, 2016.

[11] Z. Wu and H. Leng, "Complex hybrid projective synchronization of complex-variable dynamical networks via open-plusclosed-loop control," Journal of The Franklin Institute, vol. 354, no. 2, pp. 689-701, 2017.

[12] X. Wu, C. Xu, and J. Feng, "Complex projective synchronization in drive-response stochastic coupled networks with complexvariable systems and coupling time delays," Communications in Nonlinear Science and Numerical Simulation, vol. 20, no. 3, pp. 1004-1014, 2015.

[13] D. H. Ji, S. C. Jeong, J. H. Park, S. M. Lee, and S. C. Won, "Adaptive lag synchronization for uncertain complex dynamical network with delayed coupling," Applied Mathematics and Computation, vol. 218, no. 9, pp. 4872-4880, 2012.

[14] L. Lü, C. R. Li, L. S. Chen, and L. L. Wei, "Lag projective synchronization of a class of complex network constituted nodes with chaotic behavior," Communications in Nonlinear Science and Numerical Simulation, vol. 19, no. 8, pp. 2843-2849, 2014.

[15] F. Wang, Y. Yang, M. Hu, and X. Xu, "Projective cluster synchronization of fractional-order coupled-delay complex network via adaptive pinning control," Physica A: Statistical Mechanics and its Applications, vol. 434, pp. 134-143, 2015.

[16] Z. Tang, J. Feng, and Y. Zhao, "Global synchronization of nonlinear coupled complex dynamical networks with information exchanges at discrete-time," Neurocomputing, vol. 151, no. 3, pp. 1486-1494, 2015.

[17] X.-T. Tran and H.-J. Kang, "A novel observer-based finite-time control method for modified function projective synchronization of uncertain chaotic (hyperchaotic) systems," Nonlinear Dynamics, vol. 80, no. 1-2, pp. 905-916, 2015.

[18] H. Tirandaz and A. Karmi-Mollaee, "Modified function projective feedback control for time-delay chaotic Liu system synchronization and its application to secure image transmission," Optik - International Journal for Light and Electron Optics, vol. 147, pp. 187-196, 2017. 
[19] X. Wang and N. Wei, "Modified function projective lag synchronization of hyperchaotic complex systems with parameter perturbations and external perturbations," Journal of Vibration and Control, vol. 21, no. 16, pp. 3266-3280, 2015.

[20] J. Liu, S. Liu, and J. C. Sprott, "Adaptive complex modified hybrid function projective synchronization of different dimensional complex chaos with uncertain complex parameters," Nonlinear Dynamics, vol. 83, no. 1-2, pp. 1109-1121, 2016.

[21] F. Zhang and S. Liu, "Adaptive complex function projective synchronization of uncertain complex chaotic systems," Journal of Computational and Nonlinear Dynamics, vol. 11, no. 1, Article ID 011013, 2016.

[22] H. Du, "Function projective synchronization in complex dynamical networks with or without external disturbances via error feedback control," Neurocomputing, vol. 173, pp. 14431449, 2016.

[23] X. Wu and H. Lu, "Generalized function projective (lag, anticipated and complete) synchronization between two different complex networks with nonidentical nodes," Communications in Nonlinear Science and Numerical Simulation, vol. 17, no. 7, pp. 3005-3021, 2012.

[24] S.-G. Wang and S. Zheng, "Adaptive function projective synchronization of uncertain complex dynamical networks with disturbance," Chinese Physics B, vol. 22, no. 7, Article ID 070503, 2013.

[25] S. Wang, S. Zheng, B. Zhang, and H. Cao, "Modified function projective lag synchronization of uncertain complex networks with time-varying coupling strength," Optik - International Journal for Light and Electron Optics, vol. 127, no. 11, pp. 47164725, 2016.

[26] H. Du, P. Shi, and N. Lü, "Function projective synchronization in complex dynamical networks with time delay via hybrid feedback control," Nonlinear Analysis: Real World Applications, vol. 14, no. 2, pp. 1182-1190, 2013.

[27] Y. Liang and X. Y. Wang, "Chaotic synchronization in complex networks with delay nodes by non-delay and delay couplings," Acta Physica Sinica, vol. 62, no. 1, Article ID 018901, 2013.

[28] H. Zhang, M. Zhao, Z. Wang, and Z. Wu, "Adaptive synchronization of an uncertain coupling complex network with timedelay," Nonlinear Dynamics, vol. 77, no. 3, pp. 643-653, 2014.

[29] Y. Zhang, W. Yan, and Q. Yang, "Synchronization control of time-varying complex dynamic network with nonidentical nodes and coupling time-delay," Mathematical Problems in Engineering, vol. 2014, Article ID 461635, 8 pages, 2014. 


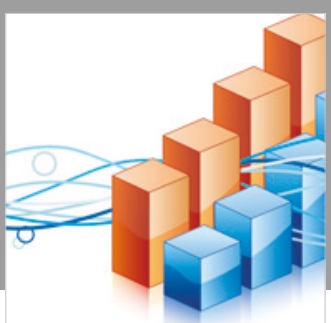

Advances in

Operations Research

\section{-n-m}
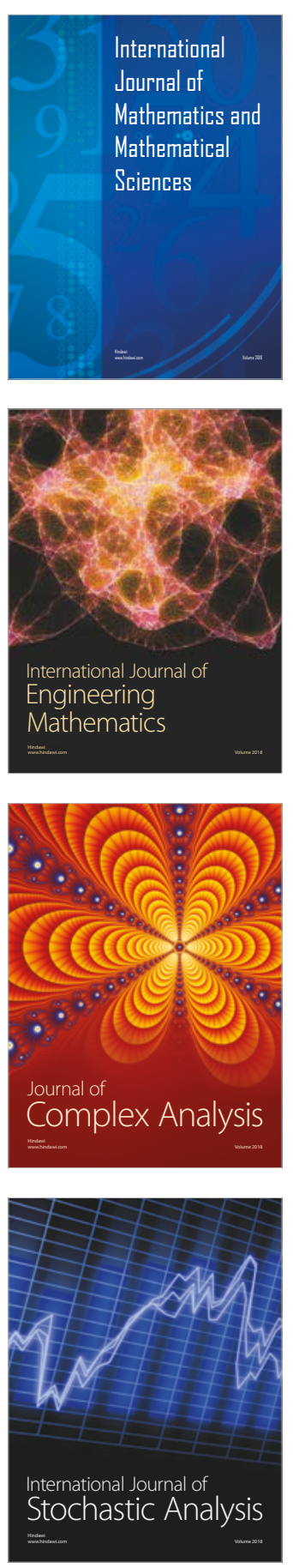
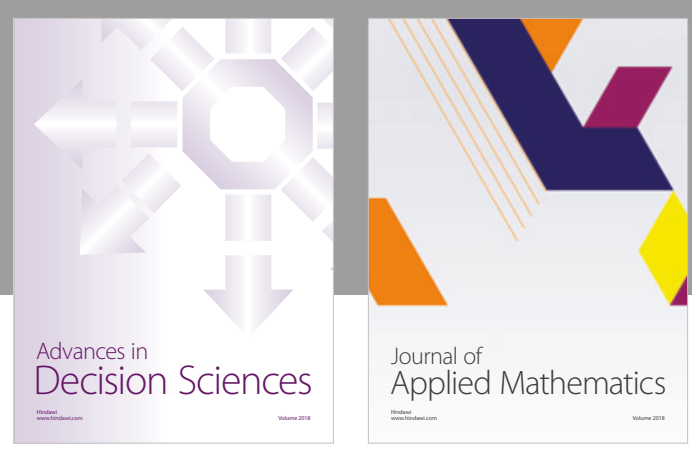

Journal of

Applied Mathematics
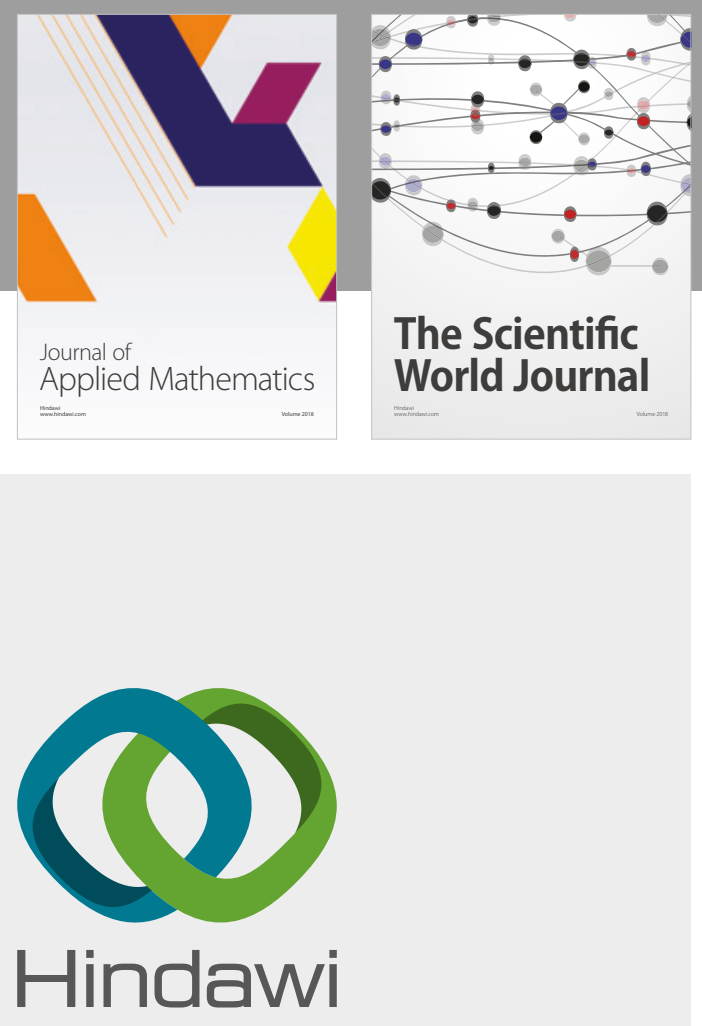

Submit your manuscripts at

www.hindawi.com

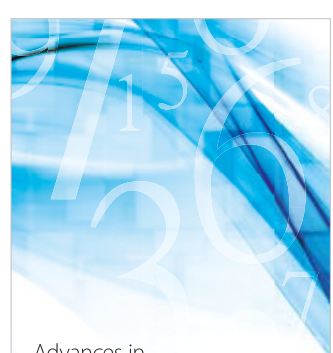

Advances in
Numerical Analysis
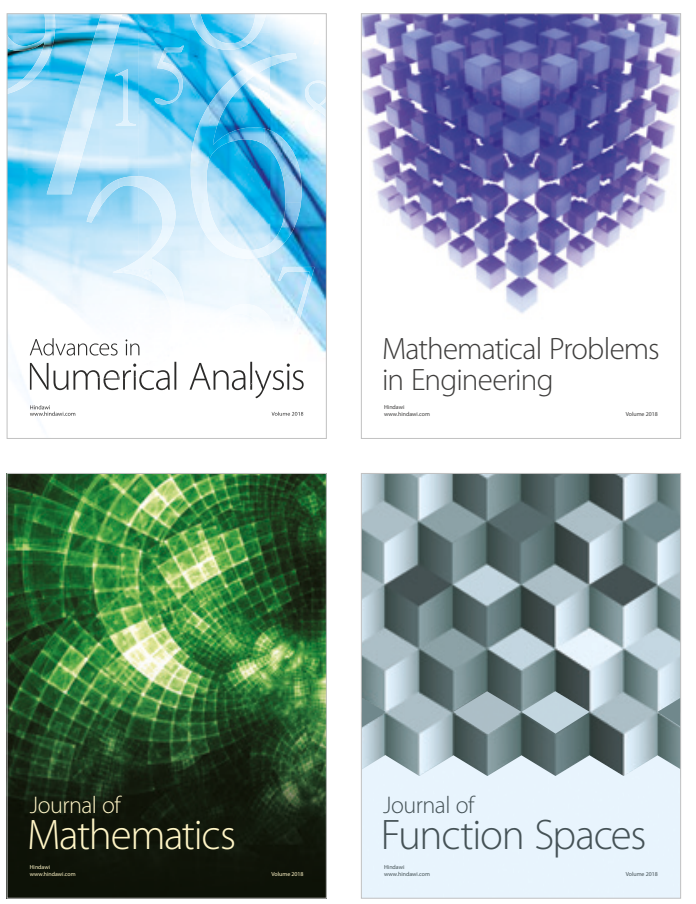

Mathematical Problems in Engineering

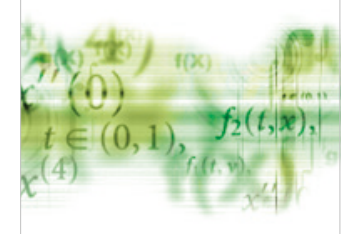

International Journal of

Differential Equations

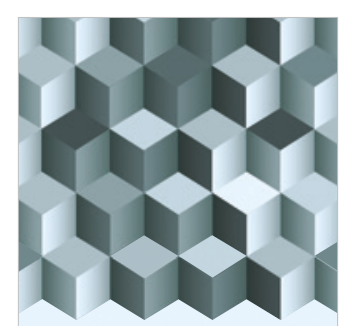

Journal of

Function Spaces

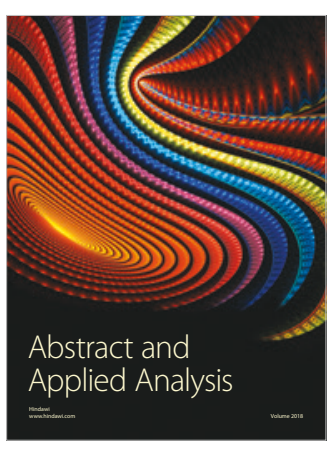

The Scientific

World Journal

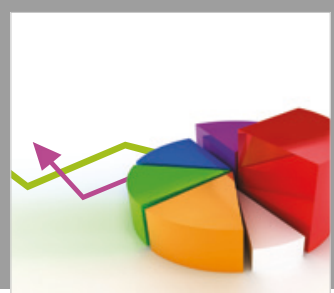

Journal of

Probability and Statistics
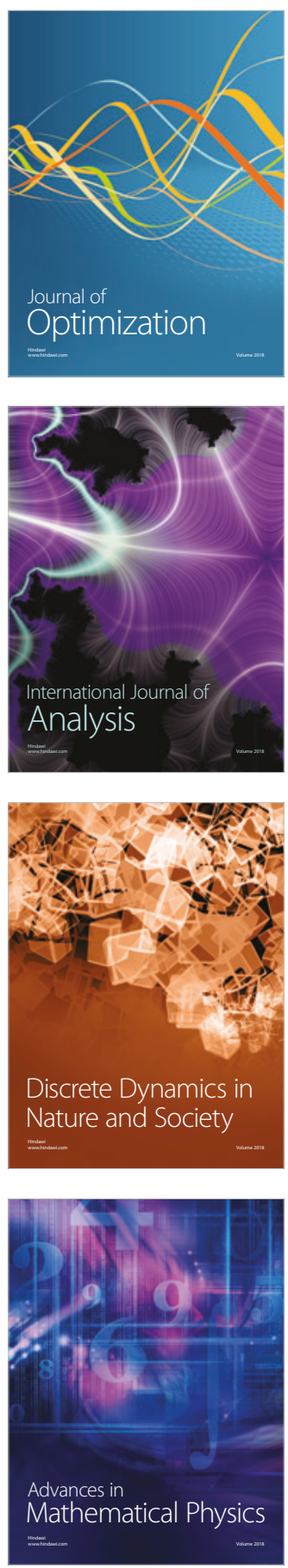